\title{
Marino-Vafa 公式的一个注记
}

\author{
卢文轩*
}

(清华大学数学科学系, 北京 100084)

\begin{abstract}
摘要 曲线模空间上的 Hodge 积分自然地出现在使用局部化方法计算 GromovWitten 不变量的过程中. Marino-Vafa 的一个惊人的公式将 Hodge 积分的一个生 成函数表示为一些看起来和原本是代数几何对象的 Hodge 积分无关的组合和代 数的量. 本文将公式直接展开并仔细估计所涉及的项, 最终证明除了一个特定类 型, 其余至多包含 3 个 Hodge 类的 Hodge 积分都可以由 Marino-Vafa 公式计算. 这意味着大量有关模空间和 Gromov-Witten 不变量的信息包含在这一复杂的公式 中. 给出了一些低亏格的例子, 这些例子与文献中的已有结果吻合. 在承认关于 Hodge 类的约化的 Mumford 关系的前提下证明和计算都是初等的.
\end{abstract}

\section{关键词 Hodge 积分 Gromov-Witten 不变量 Marino-Vafa 公式 Mumford 关系}

\section{1 引言}

将亏格为 $g$ 的有 $n$ 个标记点的代数曲线的模空间的 Deligne-Mumford 紧化记 为 $\bar{M}_{g, n}$. 以 $\pi: \bar{M}_{g, n+1} \rightarrow \bar{M}_{g, n}$ 记万有曲线, $\omega_{\pi}$ 记相对对偶层. Hodge 丛

$$
E=\pi_{*} \omega_{\pi}
$$

是 $\bar{M}_{g, n}$ 上的秩为 $g$ 的向量丛，以 $\lambda_{j}=c_{j}(E)$ 记 Hodge 丛的第 $j$ 陈类. 若 $s_{i}$ 是 $\pi$ 的对应于第 $i$ 个标记点的截面，则

$$
L_{i}=s_{i}^{*} \omega_{\pi}
$$

是 $\bar{M}_{g, n}$ 上的线丛，以 $\psi_{i}$ 记 $L_{i}$ 的第 1 陈类. 如下形式的积分称为 Hodge 积分:

$$
\int_{\bar{M}_{g, n}} \psi_{1}^{j_{1}} \cdots \psi_{n}^{j_{n}} \lambda_{1}^{k_{1}} \cdots \lambda_{g}^{k_{g}} .
$$

由于 $\bar{M}_{g, n}$ 的维数为 $3 g-3+n$, 故上式只有当

$$
j_{1}+\cdots+j_{n}+k_{1}+2 k_{2}+\cdots+g k_{g}=3 g-3+n
$$

*E-mail: luwenxuan@mails.tsinghua.edu.cn 
时不为 0 .

Hodge 积分在 Gromov-Witten 不变量的计算中有重要应用. 在文献 [1] 中, Marino 和 Vafa 猜测了一个关于 Hodge 积分的公式, 此公式在文献 [2] 中被证明. 令

$$
\Lambda_{g}^{\vee}(\tau)=\tau^{g}-\tau^{g-1} \lambda_{1}+\cdots+(-1)^{g} \lambda_{g}
$$

为 Hodge 丛的对偶丛的陈多项式. 对一个分拆 $\mu$,

$$
\mu_{1} \geqslant \mu_{2} \geqslant \cdots \geqslant \mu_{l(\mu)}>0,
$$

令

$$
|\mu|=\sum_{i=1}^{l(\mu)} \mu_{i}
$$

并定义

$$
\begin{aligned}
C_{g, \mu}(\tau)= & \frac{-\sqrt{-1}^{|\mu|+l(\mu)}}{|\operatorname{Aut}(\mu)|}(\tau(\tau+1))^{l(\mu)-1} \prod_{i=1}^{l(\mu)} \frac{\prod_{a=1}^{\mu_{i}-1}\left(\mu_{i}+a\right)}{\left(\mu_{i}-1\right) !} \\
& \times \int_{\bar{M}_{g, l(\mu)}} \frac{\Lambda_{g}^{\vee}(1) \Lambda_{g}^{\vee}(-\tau-1) \Lambda_{g}^{\vee}(\tau)}{\prod_{i=1}^{l(\mu)}\left(1-\mu_{i} \psi_{i}\right)} \\
& C_{\mu}(\lambda, \tau)=\sum_{g \geqslant 0} \lambda^{2 g-2+l(\mu)} C_{g, \mu}(\tau) .
\end{aligned}
$$

引入形式变量 $p=\left(p_{1}, \cdots, p_{n}, \cdots\right)$, 并对分拆 $\mu$ 定义

$$
p_{\mu}=p_{\mu_{1}} \cdots p_{\mu_{l(\mu)}},
$$

定义生成函数

$$
\begin{gathered}
C(\lambda, \tau, p)=\sum_{|\mu| \geqslant 1} C_{\mu}(\lambda, \tau) p_{\mu}, \\
C(\lambda, \tau, p)^{\bullet}=\mathrm{e}^{C(\lambda, \tau, p)} .
\end{gathered}
$$

文献 [2] 中称如下公式为 Marino-Vafa 公式:

$$
\begin{gathered}
C(\lambda, \tau, p) \\
=\sum_{n \geqslant 1} \frac{(-1)^{n-1}}{n} \sum_{\mu}\left(\sum_{\cup_{i=1}^{n} \mu^{i}=\mu} \prod_{i=1}^{n} \sum_{\left|\nu^{i}\right|=\left|\mu^{i}\right|} \frac{\chi_{\nu^{i}}\left(C\left(\mu^{i}\right)\right)}{z_{\mu^{i}}} \mathrm{e}^{\sqrt{-1}\left(\tau+\frac{1}{2}\right) \kappa_{\nu^{i}} \lambda / 2} V_{\nu^{i}}(\lambda)\right) p_{\mu} \\
C(\lambda, \tau, p)^{\bullet}=\sum_{|\mu| \geqslant 0}\left(\sum_{|\nu|=|\mu|} \frac{\chi_{\nu}(C(\mu))}{z_{\mu}} \mathrm{e}^{\sqrt{-1}\left(\tau+\frac{1}{2}\right) \kappa_{\nu} \lambda / 2} V_{\nu}(\lambda)\right) p_{\mu}
\end{gathered}
$$

其中,

$$
V_{\nu}(\lambda)=\prod_{1 \leqslant a \leqslant b \leqslant l(\mu)} \frac{\sin \left[\left(\nu_{a}-\nu_{b}+b-a\right) \lambda / 2\right]}{\sin [(b-a) \lambda / 2] \prod_{i=1}^{l(\nu)} \prod_{v=1}^{\nu_{i}} 2 \sin [(v-i+l(\nu)) \lambda / 2]},
$$


而 $\chi_{\mu}$ 是对应于 $\mu$ 的 $S_{|\mu|}$ 的不可约表示的特征标，数 $\kappa_{\mu}$ 定义为

$$
\kappa_{\mu}=|\mu|+\sum_{i}\left(\mu_{i}^{2}-2 i \mu_{i}\right),
$$

$C(\nu)$ 是 $S_{|\mu|}$ 的对应于分拆 $\nu$ 的共轭类. 对正整数 $i$, 定义

$$
m_{i}(\mu)=\left|\left\{j: \mu_{j}=i\right\}\right|,
$$

则

$$
z_{\mu}=\prod_{j} m_{j}(\mu) ! j^{m_{j}(\mu)} .
$$

将 (1) 和 (2) 式的右边分别记为 $R(\lambda, \tau, p)$ 和 $R(\lambda, \tau, p)^{\bullet}$. 文献 [2] 中证明的一 个关键之处是证明 $R(\lambda, \tau, p)$ 和 $C(\lambda, \tau, p)$ 均满足下列割 - 和 - 并 (cut-and-join) 方 程:

$$
\frac{\partial R}{\partial \tau}=\frac{\sqrt{-1} \lambda}{2} \sum_{i, j \geqslant 1}\left(i j p_{i+j} \frac{\partial^{2} R}{\partial p_{i} \partial p_{j}}+i j p_{i+j} \frac{\partial R}{\partial p_{i}} \frac{\partial R}{\partial p_{j}}+(i+j) p_{i} p_{j} \frac{\partial R}{\partial p_{i+j}}\right) .
$$

类似地, $R(\lambda, \tau, p)$ 和 $C(\lambda, \tau, p)$ 也均满足下列割 - 和 - 并方程:

$$
\frac{\partial R^{\bullet}}{\partial \tau}=\frac{\sqrt{-1} \lambda}{2} \sum_{i, j \geqslant 1}\left(i j p_{i+j} \frac{\partial^{2} R^{\bullet}}{\partial p_{i} \partial p_{j}}+(i+j) p_{i} p_{j} \frac{\partial R^{\bullet}}{\partial p_{i+j}}\right) .
$$

Marino 和 Vafa 猜测所有至多含 3 个 $\lambda$ 类的 Hodge 积分均可由 Marino-Vafa公 式计算出来. 他们计算了 $g=2, n=1$ 的情形, 得到了与已知结果吻合的结果. 本文包括如下 3 个方面的结果:

(i) 与 Marino-Vafa 期待的不同，并非所有至多含 3 个 $\lambda$ 类的 Hodge 积分均可 由 Marino-Vafa 公式计算, 因为有的积分根本不出现在公式左边. 第 1 个例子出 现在亏格为 3 时 (Marino-Vafa 只计算到亏格 2), 是

$$
\int_{\bar{M}_{3,1}} \psi_{1}^{2} \lambda_{2} \lambda_{3} \text {. }
$$

进一步地, 容易证明对一切亏格, 以下积分均不出现:

$$
\int_{\bar{M}_{g, n}} \psi_{1}^{j_{1}} \cdots \psi_{n}^{j_{n}} \lambda_{n-3} \lambda_{n-1} \lambda_{n},
$$

这里定义 $\lambda_{0}=1$. 另一方面, 我们将证明其他一切包含至多三个 Hodge 类的积分 均出现. 这一事实的几何意义尚不清楚.

(ii) 证明了在 Marino-Vafa 公式的左边几何部分出现的 Hodge 积分原则上都 可以由公式计算出来. 也就是说，除了一类例外情形，所有至多含 3 个 $\lambda$ 类的 Hodge 积分均可由 Marino-Vafa 公式计算. 要计算 $\bar{M}_{g, l(\mu)}$ 上的在 Marino-Vafa 公 式的左边几何部分出现的 Hodge 积分, 可对长度为 $l(\mu)$ 的分拆将两边展开, 并 比较系数以得到这些 Hodge 积分的方程组. 当长度为 $l(\mu)$ 的不同分拆取得充分 多时, 方程组系数矩阵必满秩, 从而可计算这些 Hodge 积分.

(iii) 计算了 $g=3, n=1$ 的 Hodge 积分并与已知结果比较. 


\section{Mumford 关系}

在文献 [3] 中, Mumford 得到以下结果, 称为 Mumford 关系:

$$
\Lambda_{g}^{\vee}(\tau) \Lambda_{g}^{\vee}(-\tau)=(-1)^{g} \tau^{2 g},
$$

将各阶的结果写出来, 即为

$$
\lambda_{k}^{2}=\sum_{i=1}^{k}(-1)^{i+1} 2 \lambda_{k-i} \lambda_{k+i} .
$$

例如

$$
\lambda_{1}^{2}=2 \lambda_{2}, \quad \lambda_{2}^{2}=2 \lambda_{1} \lambda_{3}-2 \lambda_{4}
$$

以及当亏格为 $g$ 时,

$$
\lambda_{g}^{2}=0 \text {. }
$$

在 $\Lambda_{g}^{\vee}(1) \Lambda_{g}^{\vee}(-\tau-1) \Lambda_{g}^{\vee}(\tau)$ 中, 2 个或 3 个 $\lambda$ 类的乘积中若有相同的 $\lambda$ 类, 则 可用 Mumford 关系将其化为不含相同的 $\lambda$ 类的乘积，例如

$$
\lambda_{1} \lambda_{2} \lambda_{2}=4 \lambda_{2} \lambda_{3}-2 \lambda_{1} \lambda_{4} .
$$

显然, 只需考察不含相同的 $\lambda$ 类的乘积. 下文中的 Hodge 积分均指这样的 Hodge 积分. 当给定一个 $\lambda_{i} \lambda_{j} \lambda_{k}$ 时, 乘积 $\Lambda_{g}^{\vee}(1) \Lambda_{g}^{\vee}(-\tau-1) \Lambda_{g}^{\vee}(\tau)$ 中可能有多项对其有贡 献, 如上例中的 $\lambda_{2} \lambda_{3}$. 这些贡献是本文中计算的关键, 因此现在进行详细讨论.

若 $\lambda_{i} \lambda_{j} \lambda_{k}$ 经 Mumford 关系化简后包含 $\lambda_{l} \lambda_{m} \lambda_{n}$, 且 $\lambda_{l} \lambda_{m} \lambda_{n}$ 前的系数绝对值 为 $a$, 则称 $\lambda_{i} \lambda_{j} \lambda_{k}$ 对 $\lambda_{l} \lambda_{m} \lambda_{n}$ 的贡献为 $a$. 如 $\lambda_{1} \lambda_{2} \lambda_{2}$ 对 $\lambda_{2} \lambda_{3}$ 的贡献为 4 , 对 $\lambda_{1} \lambda_{4}$ 的 贡献为 2 .

(i) $\lambda_{m}$ 的情形. $\Lambda_{g}^{\vee}(1) \Lambda_{g}^{\vee}(-\tau-1) \Lambda_{g}^{\vee}(\tau)$ 的乘积中对 $\lambda_{m}$ 有贡献的项是

$$
\lambda_{m}, \quad \lambda_{m / 2} \lambda_{m / 2}, \quad \cdots,
$$

其中第 2 项只有当 $m$ 是偶数时才有. 除了上面两项, 其他有贡献的项的贡献均不 小于 4 , 下面的讨论不需要其具体表达式. 一般地, 在贡献 $\lambda_{i} \lambda_{j} \lambda_{j}$ 中, 如果 $(i+j) / 2$ 是偶数, 则有新的贡献 $\lambda_{(i+j) / 2} \lambda_{(i+j) / 2} \lambda_{j}$, 其贡献数变为原来的 2 倍. 显然, 任何 项的贡献均形如 $2^{k}$.

(ii) $\lambda_{l} \lambda_{m} \lambda_{n}$ 的情形. 依 $l+m, m+n, l+n$ 奇偶性的不同, 贡献不超过 2 的 项可能有

$$
\lambda_{l} \lambda_{m} \lambda_{n}, \quad \lambda_{(l+m) / 2} \lambda_{(l+m) / 2} \lambda_{n}, \quad \lambda_{(l+n) / 2} \lambda_{(l+n) / 2} \lambda_{m}, \quad \lambda_{(m+n) / 2} \lambda_{(m+n) / 2} \lambda_{l} .
$$

\section{3 不出现在 Marino-Vafa 公式中的 Hodge 积分}

定理 $1 \Lambda_{g}^{\vee}(1) \Lambda_{g}^{\vee}(-\tau-1) \Lambda_{g}^{\vee}(\tau)$ 中不包含 $\lambda_{n-3} \lambda_{n-1} \lambda_{n}$, 即积分

$$
\int_{\bar{M}_{g, n}} \psi_{1}^{j_{1}} \cdots \psi_{n}^{j_{n}} \lambda_{n-3} \lambda_{n-1} \lambda_{n}
$$

不出现在 Marino-Vafa 公式中. 
证 $\Lambda_{g}^{\vee}(1) \Lambda_{g}^{\vee}(-\tau-1) \Lambda_{g}^{\vee}(\tau)$ 中对 $\lambda_{n-3} \lambda_{n-1} \lambda_{n}$ 有贡献的项是

$$
\lambda_{n-3} \lambda_{n-1} \lambda_{n}, \quad \lambda_{n-2} \lambda_{n-2} \lambda_{n}, \quad \lambda_{n-2} \lambda_{n-1} \lambda_{n-1} .
$$

$\lambda_{n-3} \lambda_{n-1} \lambda_{n}$ 的系数为

$$
\begin{aligned}
& \pm\left((-\tau-1)^{g-(n-1)} \tau^{g-n}+(-\tau-1)^{g-n} \tau^{g-(n-1)}\right. \\
& \quad+(-\tau-1)^{g-(n-3)} \tau^{g-n}+(-\tau-1)^{g-n} \tau^{g-(n-3)} \\
& \left.\quad+(-\tau-1)^{g-(n-1)} \tau^{g-(n-3)}+(-\tau-1)^{g-(n-3)} \tau^{g-(n-1)}\right),
\end{aligned}
$$

$\lambda_{n-2} \lambda_{n-2} \lambda_{n}$ 的系数为

$$
\pm\left((-\tau-1)^{g-(n-2)} \tau^{g-n}+(-\tau-1)^{g-n} \tau^{g-(n-2)}+(-\tau-1)^{g-(n-2)} \tau^{g-(n-2)}\right),
$$

$\lambda_{n-2} \lambda_{n-1} \lambda_{n-1}$ 的系数为

$$
\pm\left((-\tau-1)^{g-(n-1)} \tau^{g-(n-2)}+(-\tau-1)^{g-(n-1)} \tau^{g-(n-1)}+(-\tau-1)^{g-(n-2)} \tau^{g-(n-1)}\right) .
$$

由于 $\lambda_{n-2} \lambda_{n-2} \lambda_{n}$ 贡献 2 而 $\lambda_{n-2} \lambda_{n-1} \lambda_{n-1}$ 贡献 4, 于是 $\lambda_{n-3} \lambda_{n-1} \lambda_{n}$ 的系数为

$$
\begin{aligned}
& \pm\left((-\tau-1)^{g-(n-1)} \tau^{g-n}+(-\tau-1)^{g-n} \tau^{g-(n-1)}\right. \\
& +(-\tau-1)^{g-(n-3)} \tau^{g-n}+(-\tau-1)^{g-n} \tau^{g-(n-3)} \\
& +(-\tau-1)^{g-(n-1)} \tau^{g-(n-3)}+(-\tau-1)^{g-(n-3)} \tau^{g-(n-1)} \\
& +2(-\tau-1)^{g-(n-2)} \tau^{g-n}+2(-\tau-1)^{g-(n-2)} \tau^{g-(n-2)} \\
& +2(-\tau-1)^{g-n} \tau^{g-(n-2)}+4\left((-\tau-1)^{g-(n-1)} \tau^{g-(n-1)}\right. \\
& \left.\left.+(-\tau-1)^{g-(n-1)} \tau^{g-(n-2)}+(-\tau-1)^{g-(n-2)} \tau^{g-(n-1)}\right)\right) \\
= & \pm(-\tau-1)^{g-n} \tau^{g-n}\left(-\tau-1+\tau+(-\tau-1)^{3}+\tau^{3}+(-\tau-1) \tau^{3}\right. \\
& +(-\tau-1)^{3} \tau+2\left((-\tau-1)^{2}+(-\tau-1)^{2} \tau^{2}+\tau^{2}\right) \\
& \left.+4\left((-\tau-1) \tau+(-\tau-1) \tau^{2}+(-\tau-1)^{2} \tau\right)\right) \\
= & 0
\end{aligned}
$$

定理 2 除了 $\lambda_{n-3} \lambda_{n-1} \lambda_{n}$, 所有形如

$$
\lambda_{i} \lambda_{j} \lambda_{k} \quad(0 \leqslant i, j, k \leqslant g, i, j, k \text { 两两不等 })
$$

的类均包含在 $\Lambda_{g}^{\vee}(1) \Lambda_{g}^{\vee}(-\tau-1) \Lambda_{g}^{\vee}(\tau)$ 中. 即除了

$$
\int_{\bar{M}_{g, n}} \psi_{1}^{j_{1}} \cdots \psi_{n}^{j_{n}} \lambda_{n-3} \lambda_{n-1} \lambda_{n},
$$

其余 Hodge 积分均出现在 Marino-Vafa 公式中.

证明定理 2 需要如下 (显然的)

引理 当 $k_{1}, k_{2}, \cdots, k_{p}$ 两两不等时,

$$
(-1)^{\alpha_{1}} 2^{k_{1}}+(-1)^{\alpha_{2}} 2^{k_{2}}+\cdots+(-1)^{\alpha_{p}} 2^{k_{p}} \neq 0,
$$

这里 $k_{i}$ 和 $\alpha_{i}$ 是正整数.

定理 2 的证 (a) $\lambda_{m}$ 的情形. 
$\lambda_{m}$ 的系数为

$$
\pm\left((-\tau-1)^{g} \tau^{g}+(-\tau-1)^{g} \tau^{g-m}+(-\tau-1)^{g-m} \tau^{g}+\cdots\right),
$$

由于 $\tau^{2 g}$ 系数非零, 故上式非零.

(b) $\lambda_{l} \lambda_{m} \lambda_{n}$ 的情形.

对 $\lambda_{m} \lambda_{n}$ 有贡献的 $\lambda$ 类的乘积的系数均为形如 $(-\tau-1)^{a} \tau^{b}$ 的项之和, 其中 $(-\tau-1)$ 和 $\tau$ 的指数均不小于 $g-n$. 提出因子 $(-\tau-1)^{g-n}(\tau)^{g-n}$ 后, 将 $\lambda_{m} \lambda_{n}$ 的 系数剩下的因子记为 $f(\tau)$.

(i) $l+m$ 为奇数.

$$
\begin{aligned}
f(\tau)= \pm & \left((-\tau-1)^{n-m}+\tau^{n-m}+(-\tau-1)^{n-l}+(-\tau-1)^{n-l} \tau^{n-m}\right. \\
+ & \left.(-\tau-1)^{n-m} \tau^{n-l}+\tau^{n-l}+\cdots\right), \\
& f(1)= \pm\left(2(-2)^{n-m}+2+2(-2)^{n-l}+\cdots\right) \neq 0 .
\end{aligned}
$$

这意味着 $\lambda_{l} \lambda_{m} \lambda_{n}$ 的系数非零, 即 $\lambda_{l} \lambda_{m} \lambda_{n}$ 包含在 $\Lambda_{g}^{\vee}(1) \Lambda_{g}^{\vee}(-\tau-1) \Lambda_{g}^{\vee}(\tau)$ 中. 此 处的非零结果来自于所有除了 2 之外的项之和可被 4 整除这一事实.

(ii) $l+m$ 为偶数, $m+n$ 为偶数.

$$
\begin{aligned}
f(1)= & \pm\left(2(-2)^{n-m}+2+2(-2)^{n-l}+2(-1)^{\frac{m-l}{2}+1}\left(1+2(-2)^{n-\frac{l+m}{2}}\right)\right. \\
& +2(-1)^{\frac{n-l}{2}+1}\left((-2)^{n-m}+2(-2)^{\frac{n-l}{2}}\right) \\
& \left.+2(-1)^{\frac{n-m}{2}+1}\left((-2)^{n-l}+2(-2)^{\frac{n-m}{2}}\right) \cdots\right) .
\end{aligned}
$$

从上一节中关于高贡献项的描述, 我们知道 $f(1)$ 形如

$$
(-1)^{\alpha_{1}} 2^{k_{1}}+(-1)^{\alpha_{2}} 2^{k_{2}}+\cdots+(-1)^{\alpha_{p}} 2^{k_{p}} .
$$

定义

$$
\begin{aligned}
f_{1}(1)= & \pm\left(2+2(-1)^{\frac{l+m}{2}+1}\left(1+2(-2)^{n-\frac{l+m}{2}}\right)+\cdots\right. \\
& \left.+2^{k}(-1)^{x_{k}}\left((-2)^{a_{k}}+2(-2)^{b_{k}}\right)+\cdots\right),
\end{aligned}
$$

即 $f_{1}(1)$ 中除了 2 以外的所有项之和正好是可以由 Mumford 关系变为 $\lambda_{(l+m) / 2}$ $\cdot \lambda_{(l+m) / 2} \lambda_{n}$ (并最终变为 $\lambda_{l} \lambda_{m} \lambda_{n}$ ) 的项之和. 类似地, 对应于 $\lambda_{(l+n) / 2} \lambda_{(l+n) / 2} \lambda_{m}$, 定义

$$
f_{2}(1)= \pm\left(2(-2)^{n-m}+2(-1)^{\frac{n-l}{2}+1}\left((-2)^{n-m}+2(-2)^{\frac{n-l}{2}}\right)+\cdots\right),
$$

对应于 $\lambda_{(m+n) / 2} \lambda_{(m+n) / 2} \lambda_{l}$, 定义

$$
f_{3}(1)= \pm\left(2(-2)^{n-l}+2(-1)^{\frac{n-m}{2}+1}\left((-2)^{n-l}+2(-2)^{\frac{n-m}{2}}\right)+\cdots\right) .
$$

现在我们发现如下关键的抵消论据: 如果 $\lambda_{i} \lambda_{i} \lambda_{j}$ 对 $f_{1}(1)$ 贡献

$$
\pm 2^{k}\left((-2)^{n-j}+2(-2)^{n-i}\right),
$$

则 $\lambda_{i} \lambda_{(i+j) / 2} \lambda_{(i+j) / 2}$ (如果它存在) 贡献

$$
\pm 2^{k+1}\left((-2)^{n-i}+2(-2)^{n-\frac{i+j}{2}}\right)
$$


或

$$
\mp 2^{k+1}\left((-2)^{n-i}+2(-2)^{n-\frac{i+j}{2}}\right),
$$

而且 $\lambda_{i} \lambda_{(i+j) / 2} \lambda_{(i+j) / 2}$ 不是最后一项 (即 $\frac{i+j}{2}+i$ 是偶数) 当且仅当 $2^{k} 2(-2)^{n-i}$ 和 $2^{k+1}(-2)^{n-i}$ 相互抵消. $\lambda_{i} \lambda_{i} \lambda_{j}$ 的情形是类似的. 通过这个论据立刻看出 $f_{t}(1)(t=$ $1,2,3)$ 形如

$$
\pm 2^{k_{t}}\left((-2)^{a_{k_{t}}}+(-2)^{b_{k_{t}}}\right) .
$$

考虑到关系式

$$
a_{k_{1}}+2 b_{k_{1}}=a_{k_{2}}+2 b_{k_{2}}=a_{k_{3}}+2 b_{k_{3}},
$$

不难证明

$$
f(1)=f_{1}(1)+f_{2}(1)+f_{3}(1)
$$

具有引理中的形式, 于是它不为零.

(iii) $l+m$ 为偶数, $m+n$ 为奇数.

$$
f(1)= \pm\left(2(-2)^{n-m}+2+2(-2)^{n-l}+2(-1)^{\frac{m-l}{2}+1}\left(1+2(-2)^{n-\frac{l+m}{2}}\right)+\cdots\right)
$$

除了 $2+2(-1)^{\frac{m-l}{2}+1}$ 之外的所有项之和 $S$ 可被 4 整除而且 (通过上面的抵消论 据) 非零, 所以 $f(1)=0$ 仅当 $S=-4$. 这意味着

$$
n-m=1 \quad\left(\text { 即 } 2(-2)^{n-m}=-4\right) \text {, }
$$

且剩余的求和为零. 不难看出, 如果 $l<n-3$ (注意 $l \leqslant n-3) 2(-2)^{n-l}$ 的指数 将太大以致于该项不能被 $2(-1)^{\frac{m-l}{2}+1} 2(-2)^{n-\frac{l+m}{2}}$ 抵消. 由抵消论据知道

$$
S-2(-2)^{n-m} \neq 0 \text {, }
$$

而这是矛盾的, 于是 $\lambda_{n-3} \lambda_{n-1} \lambda_{n}$ 是唯一可能的情形, 我们已在定理 1 中证明其 系数确为零.

证毕.

\section{4 计算 Hodge 积分}

本节描述一个计算所有出现在 Marino-Vafa 公式左边的 Hodge 积分的算法. 对每一个长度为 $l(\mu)=n$ 的分拆 $\mu$, 将 (1) 式的左边和右边展为 $\lambda$ 的级数, 比较 $\lambda^{2 g-2+n}$ 的系数便得到一个两边是关于 $\tau$ 的多项式的恒等式. 此恒等式成立意味 着等式两边 $\tau$ 的各次项系数对应相等, 而这些系数正是 Hodge 积分的线性组合, 于是得到若干关于 $\bar{M}_{g, n}$ 上的 Hodge 积分的线性方程. 若能证明当不同分拆取得 充分多时, 方程组系数矩阵必满秩, 则可计算出这些 Hodge 积分. 在将右边展为 $\lambda$ 的级数方面, 除使用原先的表达式外还有其他较简单的方法. Zhou ${ }^{1)}$ 利用割 和 - 并方程和 “初条件”

$$
C(\lambda, 0, p)=R(\lambda, 0, p)=-\sum_{d \geqslant 1} \frac{\sqrt{-1}^{d+1} p_{d}}{2 d \sin (d \lambda / 2)},
$$

1) Zhou J. Hodge integrals, Hurwitz numbers, and symmetric groups. math.AG/0308024 
可递归地给出 $C(\lambda, \tau, p)$ 的下列表达式:

$$
\begin{aligned}
C(\lambda, \tau, p)=1 & +\frac{p_{1}}{2 \sin (\lambda / 2)}+p_{2} \frac{\sqrt{-1} \sin [(\tau+1 / 2) \lambda]}{4 \sin (\lambda / 2) \sin \lambda} \\
& -p_{1}^{2} \frac{\sin (\tau \lambda / 2) \sin [(\tau+1) \lambda / 2]}{4 \sin (\lambda / 2) \sin \lambda}+\cdots .
\end{aligned}
$$

利用

$$
\frac{t / 2}{\sin (t / 2)}=1+\sum_{g \geqslant 1} \frac{2^{2 g-1}-1}{2^{2 g-1}} \frac{\left|B_{2 g}\right|}{(2 g) !} t^{2 g}
$$

可方便地将 $C(\lambda, \tau, p)$ 展开.

下面证明当长度为 $l(\mu)=n$ 的不同拆分取得充分多时，方程组系数矩阵必 满秩.

设 $\bar{M}_{g, n}$ 上的出现在 Marino-Vafa 公式左边的 Hodge 积分有 $p$ 个:

$$
\left(h_{1}, h_{2}, \cdots, h_{p}\right),
$$

其中

$$
h_{i}=\int_{\bar{M}_{g, n}} \psi_{1}^{d_{1 i}} \cdots \psi_{n}^{d_{n i}} \lambda_{1}^{D_{1 i}} \cdots \lambda_{g}^{D_{g i}},
$$

这里 $D$ 为 1 或 0 . 再设当长度为 $n$ 的分拆取为 $(1,1, \cdots, 1)$ 时, 我们得到关于这些 Hodge 积分的系数矩阵秩为 $q$ 的方程组, 即

$$
\left(\begin{array}{cccc}
a_{11} & a_{12} & \cdots & a_{1 p} \\
a_{21} & a_{22} & \cdots & a_{2 p} \\
\vdots & \vdots & \ddots & \vdots \\
a_{q 1} & a_{q 2} & \cdots & a_{q p}
\end{array}\right)\left(\begin{array}{c}
h_{1} \\
h_{2} \\
\vdots \\
h_{p}
\end{array}\right)=\left(\begin{array}{c}
b_{1} \\
b_{2} \\
\vdots \\
b_{q}
\end{array}\right) .
$$

显然 $q \leqslant p$. 若 $q=p$, 则系数矩阵已满秩. 现设 $q<p$, 因为行秩等于列秩, 于是系 数矩阵的列向量组的秩为 $q$. 不妨设前 $q$ 列线性无关，则当 $k>q$ 时，

$$
A_{k}=\sum_{i=1}^{q} c_{i} A_{i},
$$

其中 $A_{i}$ 是 (7) 式中矩阵的第 $i$ 个列向量. 现取一个长度为 $n$ 的分拆 $\mu$,

$$
\mu_{1} \geqslant \mu_{2} \geqslant \cdots \geqslant \mu_{n}>0,
$$

我们有一些新的方程

$$
\left(\begin{array}{cccc}
a_{11} \prod_{j=1}^{n} \mu_{j}^{d_{j 1}} & a_{12} \prod_{j=1}^{n} \mu_{j}^{d_{j 2}} & \cdots & a_{1 p} \prod_{j=1}^{n} \mu_{j}^{d_{j p}} \\
a_{21} \prod_{j=1}^{n} \mu_{j}^{d_{j 1}} & a_{22} \prod_{j=1}^{n} \mu_{j}^{d_{j 2}} & \cdots & a_{2 p} \prod_{j=1}^{n} \mu_{j}^{d_{j p}} \\
\vdots & \vdots & \ddots & \vdots \\
a_{q 1} \prod_{j=1}^{n} \mu_{j}^{d_{j 1}} & a_{q 2} \prod_{j=1}^{n} \mu_{j}^{d_{j 2}} & \cdots & a_{q p} \prod_{j=1}^{n} \mu_{j}^{d_{j p}}
\end{array}\right)\left(\begin{array}{c}
h_{1} \\
h_{2} \\
\vdots \\
h_{p}
\end{array}\right)=\left(\begin{array}{c}
b_{1}^{1} \\
b_{2}^{1} \\
\vdots \\
b_{q}^{1}
\end{array}\right) .
$$


下面将说明

$$
\left(\begin{array}{cccc}
A_{1} & A_{2} & \cdots & A_{p} \\
A_{1} \prod_{j=1}^{n} \mu_{j}^{d_{j 1}} & A_{2} \prod_{j=1}^{n} \mu_{j}^{d_{j 2}} & \cdots & A_{p} \prod_{j=1}^{n} \mu_{j}^{d_{j p}}
\end{array}\right)
$$

的秩至少为 $q+1$. 只需说明第 $k(k>q)$ 个列向量与前 $q$ 个线性无关. 若不然, 考 虑到 (8) 式, 必有

$$
\left(\begin{array}{c}
A_{k} \\
A_{k} \prod_{j=1}^{n} \mu_{j}^{d_{j k}}
\end{array}\right)=\sum_{i=1}^{q} c_{i}\left(\begin{array}{c}
A_{i} \\
A_{i} \prod_{j=1}^{n} \mu_{j}^{d_{j i}}
\end{array}\right) .
$$

将 (11) 式两边矩阵中的后 $q$ 行同除以 $\prod_{j=1}^{n} \mu_{j}^{d_{j k}}$, 然后减去前 $q$ 行, 得到

$$
0=\sum_{i=1}^{q} c_{i}\left(\frac{\prod_{j=1}^{n} \mu_{j}^{d_{j i}}}{\prod_{j=1}^{n} \mu_{j}^{d_{j k}}}-1\right) A_{i} .
$$

由 $A_{i}(1 \leqslant i \leqslant q)$ 的线性相关性, 上式中系数为 0 . 即当 $c_{i} \neq 0$ 时,

$$
\prod_{j=1}^{n} \mu_{j}^{d_{j i}}=\prod_{j=1}^{n} \mu_{j}^{d_{j k}}
$$

由 $\mu_{j}$ 的任意性, 我们有

$$
d_{j k}=d_{j i},
$$

即

$$
h_{k}=\int_{\bar{M}_{g, n}} \psi_{1}^{d_{1 i}} \cdots \psi_{n}^{d_{n i}} \lambda_{1}^{D_{1 k}} \cdots \lambda_{g}^{D_{g k}} .
$$

显然

$$
D_{1 i}+2 D_{2 i}+\cdots+g D_{g i}=D_{1 k}+2 D_{2 k}+\cdots+g D_{g k} .
$$

由于 $A_{k}$ 可表为与满足 (14) 式的 $h_{i}$ 相乘的 $A_{i}$ 的线性组合, 所以在 $\Lambda_{g}^{\vee}(1) \Lambda_{g}^{\vee}(-\tau-$ 1) $\Lambda_{g}^{\vee}(\tau)$ 中, 当 $i+j+k$ 给定时, 不同的 $\lambda_{i} \lambda_{j} \lambda_{k}$ 的系数 ( $\tau$ 的多项式) 只差一个常 数. 此时我们只能计算 $i+j+k$ 给定的不同的 $\lambda_{i} \lambda_{j} \lambda_{k}$ 的线性组合的值, 而不能 计算 $\lambda_{i} \lambda_{j} \lambda_{k}$. 但利用第 2 节中的分类, 将 $\lambda_{i} \lambda_{j} \lambda_{k}$ 的系数写出并展开, 便容易看出 这是不可能的. 这样, 矩阵 (10) 的秩至少为 $q+1$. 再取一个新的分拆 $\nu$,

$$
\nu_{1} \geqslant \nu_{2} \geqslant \cdots \geqslant \nu_{n}>0,
$$

类似地可证明以下矩阵的秩至少为 $q+2$ :

$$
\left(\begin{array}{cccc}
A_{1} & A_{2} & \cdots & A_{p} \\
A_{1} \prod_{j=1}^{n} \mu_{j}^{d_{j 1}} & A_{2} \prod_{j=1}^{n} \mu_{j}^{d_{j 2}} & \cdots & A_{p} \prod_{j=1}^{n} \mu_{j}^{d_{j p}} \\
A_{1} \prod_{j=1}^{n} \nu_{j}^{d_{j 1}} & A_{2} \prod_{j=1}^{n} \nu_{j}^{d_{j 2}} & \cdots & A_{p} \prod_{j=1}^{n} \nu_{j}^{d_{j p}}
\end{array}\right) .
$$


重复以上过程, 每选取一个新的分拆, 关于 Hodge 积分的方程组的系数矩阵秩 至少增加 1 , 直至矩阵满秩. 于是当长度为 $l(\mu)$ 的不同分拆取得充分多时, 在 Marino-Vafa 公式的左边出现的 Hodge 积分原则上都可以由公式计算出来.

另外, Marino-Vafa 公式虽不能计算不出现在公式中的 Hodge 积分

$$
\int_{\bar{M}_{g, n}} \psi_{1}^{j_{1}} \cdots \psi_{n}^{j_{n}} \lambda_{2} \lambda_{3},
$$

但可利用文献 [4] 中的结果来计算 (某些) 这类 Hodge 积分.

文献 [4] 导出了下列公式:

$$
1+\sum_{g>0} \lambda^{2 g} \int_{\bar{M}_{g, 1}} \frac{\Lambda_{g}^{\vee}(1) \Lambda_{g}^{\vee}(\alpha)}{1-\psi_{0}}=\exp \left(-\frac{\lambda^{2}}{24}\right)
$$

和

$$
\int_{\bar{M}_{g, n+1}} \prod_{i=1}^{n} \psi_{i}^{a_{i}} \frac{\Lambda_{g}^{\vee}(1) \Lambda_{g}^{\vee}(\alpha)}{1-\psi_{0}}=0, \quad|a|<g,
$$

以及

$$
\begin{aligned}
& \sum_{g \geqslant 0} \lambda^{2 g} \sum_{n \geqslant 0} \sum_{|a|=g} \frac{y_{a_{1}} \cdots y_{a_{n}}}{n !} \int_{\bar{M}_{g, n+1}} \prod_{i=1}^{n} \psi_{i}^{a_{i}} \frac{\Lambda_{g}^{\vee}(1) \Lambda_{g}^{\vee}(\alpha)}{1-\psi_{0}} \\
= & \exp \left(y_{0}+\sum_{g>0} \lambda^{g} y_{g} \int_{\bar{M}_{g, 2}} \frac{\left(-\psi_{1}\right)^{g}}{1-\psi_{0}}\right) .
\end{aligned}
$$

式中 $\psi_{0}$ 是 $\bar{M}_{g, 1}$ 上的类 (本文中其他部分用 $\psi_{1}$ 表示此类), $\alpha$ 是任意复数,

$$
|a|=\sum_{i=1}^{n} a_{i},
$$

当 $|a| \leqslant g$ 时, 可用 (17) 和 (18) 式计算

$$
\int_{\bar{M}_{g, n+1}} \psi_{1}^{a_{1}} \cdots \psi_{n}^{a_{n}} \frac{\lambda_{2} \lambda_{3}}{1-\psi_{0}} .
$$

要计算

$$
\int_{\bar{M}_{g, 1}} \psi_{1}^{k} \lambda_{2} \lambda_{3}
$$

则可用 (16) 式.

\section{$5 g=3, n=1$ 的情形}

$$
\begin{aligned}
& g=3, n=1 \text { 时, 可用 } d=|\mu| \text { 来标记分拆 } \mu \text { (因为 } l(\mu)=n=1) . \\
& \int_{\bar{M}_{3,1}} \frac{\Lambda_{3}^{\vee}(1) \Lambda_{3}^{\vee}(-\tau-1) \Lambda_{3}^{\vee}(\tau)}{1-d \psi_{1}} \\
= & \left(d^{6} \int_{\bar{M}_{3,1}} \lambda_{1} \psi_{1}^{6}+d^{4} \int_{\bar{M}_{3,1}} \lambda_{3} \psi_{1}^{4}-d^{5} \int_{\bar{M}_{3,1}} \lambda_{2} \psi_{1}^{5}-d^{7} \int_{\bar{M}_{3,1}} \psi_{1}^{7}\right) \tau^{6}
\end{aligned}
$$




$$
\begin{aligned}
& +3\left(d^{6} \int_{\bar{M}_{3,1}} \lambda_{1} \psi_{1}^{6}+d^{4} \int_{\bar{M}_{3,1}} \lambda_{3} \psi_{1}^{4}-d^{5} \int_{\bar{M}_{3,1}} \lambda_{2} \psi_{1}^{5}-d^{7} \int_{\bar{M}_{3,1}} \psi_{1}^{7}\right) \tau^{5} \\
& +\left(4 d^{6} \int_{\bar{M}_{3,1}} \lambda_{1} \psi_{1}^{6}-5 d^{5} \int_{\bar{M}_{3,1}} \lambda_{2} \psi_{1}^{5}+3 d^{4} \int_{\bar{M}_{3,1}} \lambda_{3} \psi_{1}^{4}\right. \\
& \left.+d^{4} \int_{\bar{M}_{3,1}} \lambda_{1} \lambda_{2} \psi_{1}^{4}-d^{3} \int_{\bar{M}_{3,1}} \lambda_{1} \lambda_{3} \psi_{1}^{3}-3 d^{7} \int_{\bar{M}_{3,1}} \psi_{1}^{7}\right) \tau^{4} \\
& +\left(3 d^{6} \int_{\bar{M}_{3,1}} \lambda_{1} \psi_{1}^{6}-5 d^{5} \int_{\bar{M}_{3,1}} \lambda_{2} \psi_{1}^{5}+d^{4} \int_{\bar{M}_{3,1}} \lambda_{3} \psi_{1}^{4}\right. \\
& \left.+2 d^{4} \int_{\bar{M}_{3,1}} \lambda_{1} \lambda_{2} \psi_{1}^{4}-2 d^{3} \int_{\bar{M}_{3,1}} \lambda_{1} \lambda_{3} \psi_{1}^{3}-d^{7} \int_{\bar{M}_{3,1}} \psi_{1}^{7}\right) \tau^{3} \\
& +\left(d^{6} \int_{\bar{M}_{3,1}} \lambda_{1} \psi_{1}^{6}-3 d^{5} \int_{\bar{M}_{3,1}} \lambda_{2} \psi_{1}^{5}+3 d^{4} \int_{\bar{M}_{3,1}} \lambda_{3} \psi_{1}^{4}\right. \\
& \left.+d^{4} \int_{\bar{M}_{3,1}} \lambda_{1} \lambda_{2} \psi_{1}^{4}-2 d^{3} \int_{\bar{M}_{3,1}} \lambda_{1} \lambda_{3} \psi_{1}^{3}+d \int_{\bar{M}_{3,1}} \lambda_{1} \lambda_{2} \lambda_{3} \psi_{1}\right) \tau^{2} \\
& +\left(-d^{5} \int_{\bar{M}_{3,1}} \lambda_{2} \psi_{1}^{5}+3 d^{4} \int_{\bar{M}_{3,1}} \lambda_{3} \psi_{1}^{4}-d^{3} \int_{\bar{M}_{3,1}} \lambda_{1} \lambda_{3} \psi_{1}^{3}+d \int_{\bar{M}_{3,1}} \lambda_{1} \lambda_{2} \lambda_{3} \psi_{1}\right) \tau \\
& +d^{4} \int_{\bar{M}_{3,1}} \lambda_{3} \psi_{1}^{4} \text {. }
\end{aligned}
$$

将 (6) 式右边的 $p_{1}, p_{2}, \cdots$ 项 (分别对应于 $\left.d=1,2, \cdots\right)$ 对 $\lambda$ 展开并比较 $\lambda^{5}(5=$ $2 g-2+n)$ 的系数, 便可算出

$$
\begin{gathered}
\int_{\bar{M}_{3,1}} \psi_{1}^{7}=\frac{1}{82944}, \quad \int_{\bar{M}_{3,1}} \lambda_{1} \psi_{1}^{6}=\frac{7}{138240}, \\
\int_{\bar{M}_{3,1}} \lambda_{2} \psi_{1}^{5}=\frac{41}{580608}, \quad \int_{\bar{M}_{3,1}} \lambda_{3} \psi_{1}^{4}=\frac{31}{967680}, \\
\int_{\bar{M}_{3,1}} \lambda_{1} \lambda_{2} \psi_{1}^{4}=\frac{23}{193536}, \quad \int_{\bar{M}_{3,1}} \lambda_{1} \lambda_{3} \psi_{1}^{3}=\frac{41}{1451520}, \\
\int_{\bar{M}_{3,1}} \lambda_{1} \lambda_{2} \lambda_{3} \psi_{1}=\frac{1}{362880} .
\end{gathered}
$$

在 (16) 式中令 $\alpha=1$, 再将上面的结果代入, 得

$$
\int_{\bar{M}_{3,1}} \lambda_{2} \lambda_{3} \psi_{1}^{2}=\frac{1}{120960} .
$$

上面的结果和文献 [5] 中证明的 $\lambda_{g}$ 猜想以及文献 [6,7] 中得到的含 $\lambda_{g-1}$ 或 $\lambda_{g} \lambda_{g-1} \lambda_{g-2}$ 的 Hodge 积分等式吻合.

\section{参考文献}

1 Marino M, Vafa C. Framed knots at large N. In: Adem A, Morava J, Ruan Y B, eds. Orbifolds in

Mathematics and Physics: Proceedings of a Conference on Orbifolds in Mathematics and Physics, May 4-8, 2001, Madison, Wisconsin. Contemp Math, Vol 310. Providence: Amer Math Soc, 2002. 185 204 
2 Liu C, Liu K, Zhou J. A proof of a conjecture of Marino-Vafa on Hodge integrals. J Differential Geom, 2003, 65(2): 289 340

3 Mumford D. Towards an enumerative geometry of the moduli space of curves. In: Artin M, Tate J, eds. Arithmetic and Geometry: Papers Dedicated to I. R. Shafarevich on the Occasion of His Sixtieth Birthday, Vol 2. Boston: Birkhäuser, 1983. 271 326

4 Tian G, Zhou J. Quadratic recursion relations of Hodge integrals via localization. Acta Mathematica Sinica, English Series, 2003, 19(2): 209 232

5 Faber C, Pandharipande R. Hodge integrals, partition matrices, and the $\lambda_{g}$ conjecture. Ann of Math, (2), 2003, 157(1): 97 124

6 Getzler E. Okounkov A, Pandharipande R. Multipoint series of Gromov-Witten invariants of $\mathrm{CP}^{1}$. Lett Math Phys, 2002, 62(2): 159 170

7 Faber C, Pandharipande R. Hodge integrals and Gromov-Witten theory. Invent Math, 2000, 139(1): $173 \sim 199$ 